\title{
O "Terceiro Setor na Saúde": temas de investigação, atores, avanços e lacunas na gestão do Sistema Único de Saúde no prisma das Ciências Sociais e Humanas em Saúde.
}

The "Third Sector in Health": research themes, actors, advances and gaps in the management of the National Health System from the perspective of Social and Human Sciences in Health.

El "Tercer Sector en Salud": temas de investigación, actores, avances y brechas en la gestión del Sistema Único de Salud desde la perspectiva de las Ciencias Sociales y Humanas en Salud.

Le "Troisième Secteur de la Santé": thèmes de recherche, acteurs, avancées et lacunes dans la gestion du Système Unifié de Santé du point de vue des Sciences Sociales et Humaines de la Santé 
1 Pesquisador Científico VI do Instituto de Saúde da Secretaria de Saúde do Estado de São Paulo, São Paulo, Brasil. Graduado em Ciências Sociais pela Pontifícia Universidade Católica de São Pulo, São Paulo, SP, Brasil, mestre em Saúde Coletiva pela Coordenadoria de Controle de Doenças da Secretaria de Saúde do Estado de São Paulo, São Paulo, Brasil. É doutorando no Programa de Pós-Graduação em Psicologia Social do Instituto de Psicologia e investigador nos Grupos de Pesquisa em Psicologia Política, Políticas Públicas e Multiculturalismo e no Núcleo de Estudos para a Prevenção da AIDS da Universidade de São Paulo, São Paulo, SP, Brasil.

E-mail: renato@isaude.sp.gov.br

${ }^{2}$ Graduada em Ciências Sociais, mestra e doutora em Ciência Ambiental e livre-docente em Ciências Sociais em Saúde pela Universidade de São Paulo, São Paulo, SP, Brasil. É Professora Associada 1 da Faculdade de Saúde Pública, sendo docente nos Programas de Pós-Graduação em Saúde Pública da Faculdade de Saúde Pública e de Pós-Graduação em Mudança Social e Participação Política da Escola de Artes, Ciências e Humanidades da Universidade de São Paulo, São Paulo, SP, Brasil.

E-mail: aureanni@usp.br

${ }^{3}$ Cientista Social e mestra em Ciências pelo Programa de Pós-Graduação em História Social da Universidade de São Paulo, São Paulo, SP, Brasil. É Pesquisadora Científica IV do Centro de Memória e Diretoria do Museu da Saúde Pública Emílio Ribas do Instituto Butantan da Secretaria da Saúde do Estado de São Paulo, São Paulo, SP, Brasil.

E-mail: olga.alves@ butantan.gov.br

4 Filósofo pela Pontifícia Universidade Católica de Minas Gerais, Belo Horizonte, MG, Brasil, mestre e doutor em Psicologia Social pela Pontifícia Universidade Católica de São Paulo, São Paulo, SP, Brasil, e Livre Docente em Economia, Gestão e Políticas Públicas na área de Sociedades Complexas, Multiculturalismo e Direitos pela Universidade de São Paulo, São Paulo, SP, Brasil. Atualmente é Professor Associado 1, docente no Bacharelado em Gestão de Políticas Públicas da Escola de Artes, Ciências e Humanidades e no Programa de PósGraduação em Psicologia Social do Instituto de Psicologia, líder do Grupo de Estudos e Pesquisas em Psicologia Política, Políticas Públicas e Multiculturalismo da Universidade de São Paulo, São Paulo, SP, Brasil.

E-mail: alessoares@usp.br 
Resumo

\section{Abstract}

Analisou-se a produção científica sobre o "Terceiro Setor na Saúde", a partir dos congressos promovidos pela Associação Brasileira de Pós Graduação em Saúde Coletiva (ABRASCO), entre 1995 e 2011, quanto à contribuição para o desenvolvimento do subcampo das Ciências Sociais e Humanas em Saúde, vinculado ao campo interdisciplinar da Saúde Coletiva. Realizou-se um estudo exploratório, descritivo, retrospectivo de abordagem quantitativa, baseado nos Anais dos cinco congressos da ABRASCO. Identificaram-se 126 resumos, classificados em 12 temas de investigação. Quase um terço dos trabalhos evidenciaram a atuação dos movimentos sociais e das ações conduzidas por Organizações Não Governamentais no setor Saúde, ao passo que os temas que abordaram o exercício do controle social nos conselhos de saúde, o mix público-privado na saúde, a gestão/gerência das Organizações Sociais da Saúde, o voluntariado e o direito constitucional à saúde foram exíguos. Recomenda-se assim, a incorporação e manutenção desses temas com baixa visibilidade na produção científica, nas agendas de pesquisa em curso nos subcampos das Ciências Sociais e Humanas em Saúde e das Políticas, Planejamento e Gestão em Saúde, considerando a sua relevância para a gestão do Sistema Único de Saúde.

Palavras-Chave: Saúde Coletiva; Ciências Sociais e Saúde; Terceiro Setor; Gestão em Saúde; Sistema Único de Saúde; Campo Científico.

The scientific production on the "Third Sector in Health" was analyzed, from the congresses promoted by the Brazilian Association of Postgraduate in Collective Health (ABRASCO), between 1995 and 2011, regarding the contribution to the development of the subfield of Social Sciences and Humanities in Health, linked to the interdisciplinary field of Collective Health. An exploratory, descriptive, retrospective study with a quantitative approach was carried out, based on the Annals of the five ABRASCO congresses. We identified 126 abstracts, classified into 12 research topics. Almost a third of the works showed the performance of social movements and the actions carried out by Non-Governmental Organizations on the Health sector, while the themes that addressed the exercise of social control in health councils, the publicprivate mix in health, the management of Social Health Organizations, the volunteering and the constitutional right to health were limited. Thus, it is recommended the incorporation and maintenance of these themes with low visibility in scientific production, in the research agendas underway in the subfields of Social Sciences and Humanities in Health and Health Policies, Planning and Management, considering its relevance to the management of the National Health System.

Keywords: Collective Health; Social and Health Sciences; Third Sector; Health Management; National Health System; Scientific Field. 
Resumen

Resumé

Nous avons analysé la production scientifique sur le "Troisième Secteur en Santé", à partir des congrès promus par l'Association Brésilienne d'Études Postgraduées en Santé Collective (ABRASCO), entre 1995 et 2011, quant à la contribution au développement du sous-domaine des Sciences Sociales et Humaines en Santé, lié au champ interdisciplinaire de la Santé Collective. Une étude exploratoire, descriptive, rétrospective avec une approche quantitative a été menée, sur la base des actes des cinq congrès ABRASCO. Nous avons identifié 126 résumés, classés en 12 thèmes de recherche. Près d'un tiers des articles témoignent de la performance des mouvements sociaux et des actions menées par les organisations non gouvernementales dans le secteur de la santé, tandis que les thèmes qui abordent l'exercice du contrôle social dans les conseils de santé, le mélange public-privé dans la santé, la gestion/le management des organisations socio-sanitaires, le volontariat et le droit constitutionnel à la santé sont rares. Ainsi, il est recommandé d'incorporer et de maintenir ces thèmes à faible visibilité dans la production scientifique, dans les agendas de recherche en cours dans les sous-domaines des sciences sociales et humaines de la santé et des politiques, planification et gestion de la santé, compte tenu de leur pertinence pour la gestion du système de santé unifié.

Mots-clés: Santé Collective; Sciences Sociales et Santé, Tiers Secteur; Gestion de la Santé; Système de Santé Unifié; Domaine Scientifique. 


\section{Introdução}

A Associação Brasileira de Pós-Graduação em Saúde Coletiva (ABRASCO), desde a sua fundação em 1979, assumiu um papel proativo no desenvolvimento e consolidação do campo da Saúde Coletiva no país. A Saúde Coletiva é considerada uma inovação brasileira, legitimada como um campo científico interdisciplinar, voltado à produção de conhecimentos e de práticas de saúde, constituído por três subáreas: a Epidemiologia, as Ciências Sociais e Humanas em Saúde, e Políticas, Planejamento e Gestão em Saúde, além da interface com a Biomedicina, a Biologia e a Estatística (Paim \& Almeida Filho, 2000).

A Saúde Coletiva ancora-se em uma concepção ampliada de saúde, não restrita à dimensão biológica, incorporando aspectos políticos e econômicos inerentes à determinação social do processo saúde-doença-cuidado. Nesse sentido, destaca-se a importância da pluralidade de atores que constituem o campo da Saúde Coletiva em estreita relação com os movimentos sociais engajados na luta pela saúde e no processo da redemocratização do país desde a década de 1980 (Ribeiro \& Castro, 2019; Ianni, 2018).

O aperfeiçoamento do campo da Saúde Coletiva contribuiu sobremaneira para a implementação do projeto da Reforma Sanitária Brasileira e vice-versa, o qual postulava um amplo processo de transformação social, não restrito ao Setor Saúde. No âmbito das políticas públicas, nos anos 1990, resultou na criação e institucionalização do Sistema Único de Saúde (SUS) à luz da promulgação da Constituição Federal de 1988. Dessa forma, a observância do direito à saúde, ratificado no artigo 196 da Carta Magna em vigência, é considerado uma conquista singular, alcançada por meio do engajamento de diversos atores, instituições e de grupos da sociedade civil organizada, que, em última instância, assegurou o acesso universal, equânime e integral à saúde, por meio da reordenação do modelo médico e hospitalocêntrico (Brasil 1990a; Brasil, 1990b; Stralen, 1996; Paim, 2000; Paim, 2008; Silva, 2015).

Nesse contexto, Jairnilson Silva Paim, argumenta que

a Saúde Coletiva, desde a sua emergência, envolve-se com lutas teóricas, paradigmática, política e ideológica, implicando repercussões na sua delimitação e renovação. Daí a relevância de revisitar o campo, a partir da sua produção científica, tecnológica, epistemológica e político-cultural. Espera-se que, além de um profícuo debate e da contribuição dos sujeitos individuais e coletivos, possam ser identificados caminhos que permitam tornar a Saúde Coletiva um espaço cada vez mais aberto a novos paradigmas diante das necessidades de saúde, dos direitos humanos, de processos emancipatórios e da democratização da vida social (Paim, 2000:146).

No Brasil, os estudos do campo das Ciências Sociais em Saúde são recentes e remontam a década de 1970 (Nunes, 2006; Minayo, 2013). Ao mapear a produção científica e as áreas de interesse, elencadas por 158 profissionais cadastrados na ABRASCO e nos eventos científicos promovidos por essa associação nos anos 1990, Canesqui (1998) identificou um conjunto de temas emergentes relacionados à sexualidade e gênero, à saúde reprodutiva, aos movimentos sociais, à educação em saúde, entre outros, demonstrando a ampliação de temáticas, bem como mudanças no interior do campo. Marsiglia e colaboradores (2003), ao pesquisarem a produção das Escolas de Ciências 
Sociais, constataram que no campo da saúde, $17 \%$ dos temas foram tipificados como "emergentes", a exemplo das temáticas abordadas nos cursos de Pós-Graduação, a partir dos anos 1990, como saúde e trabalho e qualidade de vida e ambiente.

No contexto contemporâneo é mister incorporar no subcampo das Ciências Sociais e Humanas em Saúde a abordagem de novos objetos, temas de pesquisa e perspectivas teórico-metodológicas atinentes às dinâmicas sociais, políticas e econômicas que estão em curso, considerando necessariamente, o imbricamento das relações entre a saúde e a sociedade (Ribeiro \& Castro, 2019). Ademais, as bases da construção do pensamento social em saúde que datam dos anos 1970 no Brasil, apresentam na atualidade, lacunas quanto aos modelos explicativos adotados na apreensão da complexidade dos fenômenos sociais emergentes (Ianni, 2018). Dessa forma, "as ciências sociais vêm tentando construir instrumentos conceituais e metodológicos adequados às novas realidades emergentes no mundo social. Novos temas se apresentam, metabolizados pela reflexão especializada, que passam a integrar o repertório do discurso público sobre questões sociais, ambientais, organizacionais e sanitárias" (Ribeiro \& Castro, 2019:175).

Ancorados nessa perspectiva teórica, procuramos, no presente artigo, identificar a produção científica no âmbito das Ciências Sociais e Humanas em Saúde sobre o "Terceiro Setor na Saúde", reconhecido como um tema emergente no SUS, no que tange à gestão da política e das práticas de saúde na contemporaneidade. No Setor Saúde, o chamado "Terceiro Setor", abarca um leque de organizações privadas, com finalidades e interesses públicos, atuando, sobretudo, na prestação de serviços complementares ao poder público (Barboza \& Lopes, 2009).

No lócus público, desde a década de 1990, cabe ressaltar a "Reforma do Aparelho do Estado Brasileiro", levada a cabo durante os governos do presidente Fernando Henrique Cardoso, com vistas à implantação de uma administração de caráter gerencial da coisa pública focada em resultados para superar a administração burocrática, engessada e ineficiente operada pelo Estado (Brasil, 1995; Bresser-Pereira, 2011). Um dos efeitos dessa reforma foi o acréscimo no número de organizações prestadoras de serviços, tipificadas como "Terceiro Setor", as quais passaram a atuar nas três esferas de governo, especialmente no Setor Saúde.

O crescimento do "Terceiro Setor" no Brasil, sobretudo na área da saúde foi esquadrinhado por meio dos levantamentos conduzidos pelo Instituto Brasileiro de Geografia e Estatística (IBGE) para traçar o perfil das instituições que compõem o referido setor. Em 2010, constava do Cadastro Central de Empresas do IBGE um total de 290,7 mil Fundações Privadas e Associações sem Fins Lucrativos (Fasfil), correspondendo a 5,2\% das entidades públicas e privadas, lucrativas ou não. Segundo o levantamento, 18,6\% das entidades atuavam nas áreas da Saúde, da Educação, da Pesquisa e da Assistência Social, sendo a saúde, a área com a maior oferta de empregos, perfazendo 574,5 mil trabalhadores (IBGE, 2012). Na edição de 2019, cujos dados referem-se a 2016, foram identificadas 526,8 mil entidades sem fins lucrativos num universo de 5,5 milhões de organizações e dessas 237 mil classificadas como Fasfil. Os dados revelaram que houve um crescimento de 11,7\% no contingente da força de trabalho nessas instituições, sendo mais expressivo nas entidades da área da Saúde (25,5\%), da Religião $(23,9 \%)$ e do Desenvolvimento e Defesa de Direitos $(11,4 \%)$. No caso da Saúde, foram criados 164,6 mil postos de trabalho e em conjunto com as áreas da Educação e Pesquisa, concentraram mais da metade dos trabalhadores assalariados (IBGE, 2019). Portanto, baseados nessas evidências, podemos atestar a relevância e a participação das associações e das fundações privadas no Brasil, bem como a performance junto a setores sociais estratégicos no âmbito das políticas públicas. 
Entretanto, Gohn (2000) sublinha o caráter heterogêneo das instituições que integram o "Terceiro Setor", não só quanto aos vários tipos de organizações que o compõe, mas também no que tange aos interesses e as concepções que fundamentam as práticas institucionais. Segundo a pesquisadora, o Terceiro Setor

é um tipo de 'Frankenstein': grande, heterogêneo, construído de pedaços, desajeitado, com múltiplas facetas. É contraditório, pois inclui tanto entidades progressistas como conservadoras. Abrange programas e projetos sociais que objetivam tanto a emancipação dos setores populares e a construção de uma sociedade mais justa, igualitária, com justiça social, como programas meramente assistenciais, compensatórios, estruturados segundo ações estratégico-racionais, pautadas pela lógica de mercado. Um ponto em comum: todos falam em nome da cidadania. (Gohn, 2000:60).

Corroborando os questionamentos sobre o "Terceiro Setor" no Brasil, Falconer (1999), sumariza que:

o termo terceiro setor, no uso corrente, é usado para se referir à ação social das empresas, ao trabalho voluntário de cidadãos, às organizações do poder público privatizadas na forma de fundações e 'organizações sociais'. Mais do que um conceito rigoroso ou um modelo solidamente fundamentado em teoria organizacional, política ou sociológica - terceiro setor, no Brasil, é uma ideia-força, um espaço mobilizador de reflexão, de recursos e, sobretudo, de ação. (Falconer, 1999:4).

De forma contundente, Montaño (2010) problematiza que a noção de "Terceiro Setor" é desprovida de rigor teórico e científico. O pesquisador chama a atenção que ao assumirmos esse termo, reproduzimos uma visão compartimentada e autônoma da realidade social, constituída pelo Estado, pelo mercado e pela sociedade civil. Portanto, trata-se de um equívoco, porque segundo o autor, "numa perspectiva crítica e de totalidade, este conceito resulta inteiramente ideológico e inadequado ao real" [e essa divisão] "consiste num artifício positivista, institucionalista ou estruturalista" (Montaño, 2010:182). Logo, essa segmentação de base setorial, fortalece em última instância, uma racionalidade neoliberal ao intervir nas demandas sociais, reduzindo assim, uma questão de caráter "político-econômico-ideológica numa questão meramente técnico-operativa" (Montaño, 2010:185).

Ademais, o termo "Terceiro Setor" abarca um processo de ONGuização de movimentos sociais que passam a ser capturados pelo Estado (neo)liberal e perdem parte considerável de sua autonomia para atuarem como agentes críticos frente às ações de Estados/governos que vão de encontro com a luta por justiça e mudança social que diminua as profundas desigualdades que marcam a realidade brasileira (Silva, 2007; Ferrari e cols., 2019). Esse processo afeta profundamente a qualidade e o modo de se fazer Políticas Públicas no país, reforçando modelos estadocêntricos em detrimento de modelos multicêntricos que potencializam processos participativos que valorizam a autonomia dos movimentos sociais e da sociedade civil (Barboza e cols., 2017; Godoy-Flores, 2017, 2019; Silva, 2009, 2013, 2018ab).

Nesse cenário polêmico, torna-se relevante a problematização do tema "Terceiro Setor na Saúde", com o fito de apreender as concepções adotadas na gestão, os arranjos e as práticas institucionais e, sobretudo, os seus efeitos no processo de consolidação e sustentabilidade do SUS na contemporaneidade. Assim, nosso objetivo é analisar a produção científica sobre o "Terceiro Setor na Saúde", a partir dos congressos promovidos pela ABRASCO, entre 1995 e 2011, quanto à 
contribuição para o desenvolvimento do subcampo das Ciências Sociais e Humanas em Saúde no âmbito do SUS.

\section{Percurso Metodológico}

O presente artigo é um recorte dos resultados do projeto de pesquisa "Questões Contemporâneas nas Ciências Sociais em Saúde: o estudo de temas emergentes nos Congressos Brasileiros de Ciências Sociais e Humanas em Saúde, ABRASCO 1995 - 2011", financiado pelo CNPq (Ianni e cols., 2012).

A ABRASCO promoveu, entre 1995 e 2011, cinco congressos com o fito de discutir temas prioritários e propor uma agenda de pesquisa para a institucionalização do subcampo das Ciências Sociais e Humanas em Saúde, um dos braços do campo interdisciplinar da Saúde Coletiva. Foram realizados os seguintes congressos e com os respectivos temas: o I Congresso Brasileiro de Ciências Sociais em Saúde (1995) com o tema: "Cidade e Saúde”; o II Congresso Brasileiro de Ciências Sociais em Saúde (1999) com o tema: "Ciências Sociais e Saúde: Tendências, Objetos e Abordagens"; o III Congresso Brasileiro de Ciências Sociais e Humanas em Saúde (2005) que abordou a "Ciência e Saúde Coletiva: Desafios da Fragilidade da Vida na Sociedade Contemporânea"; o IV Congresso Brasileiro de Ciências Sociais e Humanas em Saúde, X Congresso Latino-americano de Medicina Social, XIV Congress of The International Association of Health Policy (2007) com o mote: "Equidade, Ética e Direito à Saúde: Desafios à Saúde Coletiva na Mundialização"; e o V Congresso Brasileiro de Ciências Sociais e Humanas em Saúde (2011) que discutiu "O lugar das Ciências Sociais e Humanas no campo da Saúde Coletiva". Dessa forma, esses congressos demonstram a trajetória desse subcampo e constituem um lócus privilegiado para a interação e o intercâmbio da produção científica dos atores e das instituições participantes (Canesqui, 2008).

A pesquisa foi delineada como um estudo retrospectivo, exploratório-descritivo de abordagem quantitativa (Minayo, 2004). Os dados foram coletados dos Livros de Resumos dos cinco congressos referidos anteriormente. Os trabalhos científicos sobre o "Terceiro Setor na Saúde", definido como objeto do presente estudo, foram identificados, classificados e organizados em um banco de dados. Apesar das limitações desse tipo de fonte, como, por exemplo, o exíguo número de palavras, entendemos que foi possível proceder à análise do tema elencado com vistas à identificar sua relevância no subcampo das Ciências Sociais e Humanas em Saúde.

O I e o II Congressos não dispunham de CD Rom dos Anais e a seleção dos trabalhos foi efetuada por meio da leitura de cada um dos trabalhos publicados nos Anais impressos, tendo por referência as palavras-chave que cobriram o escopo do tema e orientaram a seleção dos resumos. Para o III, IV e V Congressos, a seleção foi realizada por busca ativa das palavras-chave nos $C D$ Rom e nos sites específicos dos congressos. No conjunto dos cinco congressos, foram aferidos 7.517 resumos que compõem o universo de análise da presente pesquisa. Para a seleção dos trabalhos utilizaram-se as 
palavras-chave: participação; movimentos sociais; participação social; ativismo; sociedade civil; Terceiro Setor; ONG (Organização Não Governamental); OSC (Organização da Sociedade Civil); controle social; voluntariado; rede social solidária; accountability social; rede de cooperação; rede de interesse; organizações sociais; organizações sociais da saúde; intersetorialidade; sustentabilidade e terceirização.

Todos os dados coletados foram organizados em uma planilha Excel, contendo o registro de cada resumo selecionado. Consta desse banco de dados um conjunto de variáveis intencionalmente definidas pelos pesquisadores, a saber: congresso, vínculo institucional dos autores (Academia, Serviço de Saúde/Secretaria de Saúde/Ministério da Saúde ou ONG), estado de origem da instituição, macrorregião, tipo de instituição acadêmica (Federal, Estadual, Privada), tipo de estudo (pesquisa ou relato de experiência), metodologia, modalidade de apresentação (oral, pôster, pôster eletrônico ou publicação nos Anais) e temas/objetos identificados referentes ao "Terceiro Setor na Saúde". Os dados foram submetidos à análise descritiva baseada em frequências absolutas e relativas. O projeto foi avaliado e aprovado pelo Comitê de Ética em Pesquisa do Instituto de Saúde da Secretaria de Estado da Saúde de São Paulo.

\section{Resultados e Discussão}

De acordo com a Tabela 1, no conjunto dos Anais dos cinco Congressos Brasileiros de Ciências Sociais e Humanas em Saúde realizados pela ABRASCO entre 1995 e 2011, foram publicados 7.517 trabalhos. Nesse período, contatou-se que a frequência do número de trabalhos foi crescente entre o I e o IV Congressos, sendo que esse último concentrou $42,1 \%$ da produção técnico-científica. Em relação ao tema "Terceiro Setor na Saúde" foram identificados 126 resumos, perfazendo 1,7\% do universo analisado. Esse tema foi abordado em todas as edições e ao compararmos o I e o V Congressos, verificamos que a produção aumentou 22 vezes. A proporção de trabalhos publicados foi crescente e variou de 3,2\% a 69,8\%, exceto no II Congresso que correspondeu a 1,6\% dos resumos.

Cabe destacar que os trabalhos referentes ao tema do "Terceiro Setor na Saúde" concentraram-se nas duas últimas edições, alcançando uma proporção superior a $85 \%$, provavelmente associada à maior oferta de eixos e subtemas na programação dos congressos da ABRASCO, bem como ao desenvolvimento e a maior visibilidade da participação das organizações do Terceiro Setor no âmbito da saúde pública e em outros setores sociais. O incremento contínuo observado na produção dessa temática, nos permite afirmar que provavelmente, o ponto de saturação ainda não foi alcançado no interior do subcampo investigado. Entretanto, faz-se necessário realizar o mesmo tipo de inventário nas edições subsequentes dos Congressos Brasileiros de Ciências Sociais e Humanas (VI, VII, VIII), sendo que essa última edição foi promovida pela ABRASCO em 2019 na cidade de João Pessoa. 
No que concerne a dinâmica do subcampo, o estudo conduzido por Ianni e colaboradores (2015), entrevistou os presidentes da ABRASCO e das Comissões de Ciências Sociais no período de 1995 a 2011 e identificou na análise das narrativas,

três momentos marcantes da história dos Congressos de Ciências Sociais e Humanas em Saúde: o primeiro, em 1995 quando da realização do I Congresso, que significou a maturação do conhecimento das ciências sociais em saúde, a demarcação do campo; o III Congresso, de 2005, referido como o de consolidação da área de conhecimento, consolidação desse campo científico; e o V Congresso de 2011 que pôs em pauta o lugar científico das ciências sociais e humanas em saúde no contexto da Saúde Coletiva, no Brasil (Ianni e cols., 2015:510).

Tabela 1. Frequência absoluta e relativa dos trabalhos em geral e do "Terceiro Setor na Saúde" apresentados no I, II, III, IV e V Congressos Brasileiros de Ciências Sociais e Humanas em Saúde. ABRASCO, 1995 - 2011.

\begin{tabular}{ccccc}
\hline \multirow{2}{*}{ Congresso } & \multicolumn{2}{c}{ Trabalhos } & \multicolumn{2}{c}{ Terceiro Setor } \\
& $\mathbf{N}$ & $\mathbf{\%}$ & $\mathbf{N}$ & $\mathbf{\%}$ \\
\hline I & 398 & 5,3 & 4 & 3,2 \\
II & 437 & 5,8 & 2 & 1,6 \\
III & 2032 & 27,0 & 11 & 8,7 \\
IV & 3168 & 42,1 & 21 & 16,7 \\
V & 1482 & 19,7 & 88 & 69,8 \\
\hline Total & $\mathbf{7 5 1 7}$ & $\mathbf{1 0 0}$ & $\mathbf{1 2 6}$ & $\mathbf{1 0 0}$ \\
\hline
\end{tabular}

Fonte: Anais dos Congressos ABRASCO.

A Tabela 2 mostra o ranking das temáticas relacionadas ao "Terceiro Setor na Saúde", classificadas em 12 temas e/ou objetos de investigação. Nesse conjunto, quase um terço dos trabalhos apresentados evidenciaram a atuação dos movimentos sociais e das ações conduzidas por ONGs que atuam no setor Saúde. Na sequência, observam-se os temas sobre a educação em saúde $(13,6 \%)$ e intersetorialidade e redes $(10,3 \%)$, ambos considerados no campo da Saúde Coletiva como temas clássicos e associados, sobretudo ao escopo da promoção da saúde no SUS. O tema do controle social nos conselhos de saúde aparece em seguida com 10,3\% dos trabalhos e não obstante a sua importância para a implementação das políticas públicas de saúde, o mesmo apresentou uma proporção exígua no ranking. Chama a atenção, o pequeno número de relatos de resultados de pesquisas ou de experiências com proporção inferior a 5\% coordenados pelas instituições, a respeito do voluntariado, do direito constitucional à saúde e da vigilância em saúde.

Dessa forma, ao analisarmos o ranking das temáticas sobre o "Terceiro Setor na Saúde", enfatizamos o protagonismo assumido pelas organizações da sociedade civil e pelos movimentos populares de saúde no âmbito das políticas públicas em curso no SUS. Nesse sentido, Maria da Glória Gohn, ressalta que o processo de "associativismo dos movimentos sociais, ONGs e outras entidades do Terceiro Setor", que atuam em redes no âmbito do território, operam "como agentes estruturantes de 
possíveis formas de desenvolvimento sustentável" (Gohn, 2014:86), ainda que pesem os desafios para a manutenção de projetos (Barboza e cols., 2014; Barboza e cols., 2017).

Ademais, o trabalho articulado em redes no SUS, norteia às ações de promoção da saúde, sobretudo por meio das parcerias intra e intersetoriais com instituições públicas e privadas, incluindo as organizações da sociedade civil, que apresentam potência na resposta às necessidades de saúde da população, sob a ótica dos determinantes sociais da saúde. Em última instância, esse processo de trabalho de base territorial, fortalece a capacidade de resposta dos serviços da rede de Atenção Básica, a qual deve ser o eixo estruturante do sistema de saúde, atuando em estreita interface com os demais níveis e complexidades do sistema (Buss, 2003; Heimann, Ibanhes \& Barboza, 2005; Brasil, 2011). Dessa forma, é mister identificar e intervir nas lacunas presentes no território sanitário, considerando a pluralidade de atores e instituições na perspectiva intersetorial, visando a superação da fragmentação das ações e o fortalecimento da promoção da saúde.

Tabela 2. Frequência absoluta e relativa dos temas sobre o "Terceiro Setor na Saúde" apresentados no I, II, III, IV e V Congressos Brasileiros de Ciências Sociais e Humanas em Saúde. ABRASCO, 1995 - 2011.

\begin{tabular}{lcc}
\hline \multicolumn{1}{c}{ Temas } & $\mathbf{N}^{\mathbf{0}}$ & $\mathbf{\%}$ \\
\hline Movimentos sociais e participação na saúde & 19 & 15,1 \\
Atuação de ONGs na saúde & 18 & 14,3 \\
Educação em saúde & 17 & 13,6 \\
Intersetorialidade e redes & 13 & 10,3 \\
Controle social nos conselhos de saúde & 13 & 10,3 \\
Mix público-privado & 10 & 7,9 \\
Gestão/gerência das Organizações Sociais de Saúde & 9 & 7,1 \\
Avaliação de projetos ou ações programáticas & 8 & 6,3 \\
Produção de conhecimento teórico/metodológico & 7 & 5,5 \\
Voluntariado & 6 & 4,8 \\
Direito à saúde & 5 & 4,0 \\
Vigilância em saúde & 1 & 0,8 \\
\hline Total & $\mathbf{1 2 6}$ & $\mathbf{1 0 0}$ \\
\hline
\end{tabular}

Fonte: Os autores.

A Tabela 3 apresenta a dinâmica de cada um dos temas investigados, sendo possível identificar a inclusão, a permanência ou a não incorporação de um determinado tema em cada uma das cinco edições dos congressos. Entre os temas principais do ranking, verificou-se que os resumos que versaram sobre a temática dos movimentos sociais e da participação na saúde, foram distribuídos em três edições, principalmente no I e no V Congressos. A atuação das ONGs na saúde, apareceu em quatro edições, sobretudo no III Congresso. Os resumos sobre as ações de educação em saúde, somente na última edição. O tema da intersetorialidade e redes, concentrou-se principalmente no IV Congresso e também no V Congresso, porém com menor proporção de trabalhos. Quanto ao tema do controle social nos conselhos de saúde, presente no I, IV e V Congressos, observou-se que a proporção 
dos trabalhos foi decrescente em cada uma dessas edições. Por último, os trabalhos que problematizaram os diferentes aspectos do mix público-privado na saúde foram aferidos somente no último congresso, apesar da sua relevância estratégica para a gestão do SUS.

Em grande angular, pode-se constatar que os temas identificados sobre o "Terceiro Setor na Saúde" são consonantes aos eixos definidos pelas comissões científicas dos congressos estudados. Na consulta à programação oficial dos cinco congressos, disponíveis nos livros de resumos, consta que as temáticas dos movimentos sociais e da participação na saúde foram as únicas elencadas em todas as edições, ao passo que as novas formas de gestão e gerência e a relação público-privado na saúde, foram introduzidos no II e III Congressos, respectivamente. Quanto à atuação da sociedade civil junto aos grupos específicos, sobretudo por meio das ONGs, a mesma foi incluída no III Congresso e o tema que tratou da atuação dos movimentos sociais na defesa da saúde se deu no IV Congresso. Cabe ressaltar que o último congresso teve seu formato alterado e a programação passou a ser organizada por grupos temáticos, com vistas à intensificar a interação e o intercâmbio entre os profissionais que pesquisam um mesmo tema ou objeto no subcampo das Ciências Sociais e Humanas em Saúde.

Nota-se na Tabela 3 que a introdução e a permanência dos temas sobre o "Terceiro Setor na Saúde", variou segundo a edição dos congressos. Houve incremento dos temas apresentados, variando de 3 a 11 temas, exceto no II Congresso, o qual teve somente um tema que versou sobre a atuação das ONGs na saúde. No último congresso, verificou-se uma maior diversidade de temáticas compartilhadas pelas instituições nos congressos, demonstrando assim, a complexidade dos objetos elencados nas pesquisas e nos relatos de experiências socializados. Dentre os principais temas analisados, observou-se que os resumos sobre os movimentos sociais e a participação na saúde, apresentaram uma pequena redução na proporção de trabalhos entre o primeiro e o último congresso de $25 \%$ para $19,3 \%$. Nesse período também houve diminuição nos trabalhos que abordaram a atuação das ONGs na saúde de $25 \%$ para $11,4 \%$; e sobretudo no tema do exercício do controle social nos conselhos de saúde de $50 \%$ para $6,8 \%$.

Chama a atenção, a invisibilidade de trabalhos sobre às ações de educação em saúde, considerado um tema clássico no campo da Saúde Coletiva, debatido somente no último congresso. Ademais, os temas mix público-privado na saúde e produção de conhecimento teórico-metodológico sobre o "Terceiro Setor na Saúde", ambos também só foram contemplados na última edição. Pode-se constatar que nos dois últimos congressos, realizados respectivamente em 2007 e 2011, especialmente no V Congresso, houve a introdução de novas temáticas relacionadas ao "Terceiro Setor na Saúde".

É possível inferir que esse fato está relacionado à maior diversificação e incorporação de novas disciplinas obrigatórias e eletivas no interior do campo da Saúde Coletiva, como encontrado nos achados de outros estudos, abrindo espaço para a abordagem de novos objetos de pesquisa e arranjos disciplinares e epistemológicos (Nunes e cols., 2010). Corroborando essa dinâmica, outra pesquisa demonstrou no recorte temporal entre 2002 e 2012, um incremento no número de programas de PósGraduação em Saúde Coletiva credenciados pela Coordenação de Aperfeiçoamento de Pessoal de Nível Superior (CAPES) no Ministério da Educação, passando de 23 para 40 programas acadêmicos no país, respectivamente. No período, as pesquisadoras constataram que o número total de disciplinas oferecidas mais que duplicou, de 812 para 1.793 e o número de disciplinas específicas do subcampo das Ciências Sociais e Humanas em Saúde, cresceu 1,9 vezes, passando de 169 para 322 disciplinas (Deslandes \& Maksud, 2020). 
Tabela 3. Frequência absoluta e relativa dos temas sobre o "Terceiro Setor na Saúde" apresentados nos Congressos Brasileiros de Ciências Sociais e Humanas em Saúde, segundo a edição do congresso. ABRASCO, 1995 - 2011.

\begin{tabular}{|c|c|c|c|c|c|c|c|c|c|c|c|c|}
\hline \multirow[t]{2}{*}{ Temas } & \multicolumn{2}{|r|}{ I } & \multicolumn{2}{|c|}{ II } & \multicolumn{2}{|c|}{ III } & \multicolumn{2}{|c|}{ IV } & \multicolumn{2}{|c|}{$\mathbf{V}$} & \multicolumn{2}{|c|}{ Total } \\
\hline & $\mathbf{N}$ & $\%$ & $\mathbf{N}$ & $\%$ & $\mathbf{N}$ & $\%$ & $\mathbf{N}$ & $\%$ & $\mathbf{N}$ & $\%$ & $\mathbf{N}$ & $\%$ \\
\hline Movimentos sociais e participação na saúde & 1 & 25,0 & 0 & 0,0 & 1 & 9,1 & 0 & 0,0 & 17 & 19,3 & 19 & 15,1 \\
\hline Controle social nos conselhos de saúde & 2 & 50,0 & 0 & 0,0 & 0 & 0,0 & 5 & 23,8 & 6 & 6,8 & 13 & 10,3 \\
\hline Direito à saúde & 0 & 0,0 & 0 & 0,0 & 1 & 9,1 & 0 & 0,0 & 4 & 4,5 & 5 & 4,0 \\
\hline Atuação ONGs na saúde & 1 & 25,0 & 2 & 100 & 5 & 45,5 & 0 & 0,0 & 10 & 11,4 & 18 & 14,3 \\
\hline Voluntariado & 0 & 0,0 & 0 & 0,0 & 3 & 27,3 & 1 & 4,8 & 2 & 2,3 & 6 & 4,8 \\
\hline Gestão/Gerência nas Organizações Sociais de Saúde & 0 & 0,0 & 0 & 0,0 & 1 & 9,1 & 5 & 23,8 & 3 & 3,4 & 9 & 7,1 \\
\hline Mix público-privado & 0 & 0,0 & 0 & 0,0 & 0 & 0,0 & 0 & 0,0 & 10 & 11,4 & 10 & 7,9 \\
\hline Intersetorialidade e redes & 0 & 0,0 & 0 & 0,0 & 0 & 0,0 & 8 & 38,1 & 5 & 5,7 & 13 & 10,3 \\
\hline Educação em saúde & 0 & 0,0 & 0 & 0,0 & 0 & 0,0 & 0 & 0,0 & 17 & 19,3 & 17 & 13,5 \\
\hline Avaliação de projetos ou ações programáticas & 0 & 0,0 & 0 & 0,0 & 0 & 0,0 & 1 & 4,8 & 7 & 8,0 & 8 & 6,3 \\
\hline Produção de conhecimento teórico/metodológico & 0 & 0,0 & 0 & 0,0 & 0 & 0,0 & 0 & 0,0 & 7 & 8,0 & 7 & 5,6 \\
\hline Vigilância em saúde & 0 & 0,0 & 0 & 0,0 & 0 & 0,0 & 1 & 4,8 & 0 & 0,0 & 1 & 0,8 \\
\hline Total & 4 & 100 & 2 & 100 & 11 & 100 & 21 & 100 & 88 & 100 & 126 & 100 \\
\hline
\end{tabular}

Fonte: Os autores.

$\mathrm{Na}$ Tabela 4, que apresenta o vínculo institucional do autor principal, constatou-se que majoritariamente, os trabalhos foram oriundos das instituições acadêmicas, as quais representam mais da metade da produção dos congressos examinados, alcançando 64,3\% (N=81) dos resumos. $\mathrm{Na}$ sequência, a produção dos "Serviços", que inclui a participação das unidades de saúde e das secretarias municipais e estaduais de saúde, além de equipamentos sob a gestão do Ministério da Saúde, os quais foram responsáveis por 9,5\% (N=12) dos trabalhos. Os autores vinculados à modalidade "ONGs", que contempla as associações, as fundações e outras organizações tipificadas no âmbito do "Terceiro Setor", apresentaram a menor proporção com 7,9\% (N=10) dos resumos. Nota-se que proporcionalmente, a diferença na produção dos "Serviços" e das "ONGs" foi inferior a 2\%, reafirmando, portanto, a posição hegemônica da Academia nos congressos de Ciências Sociais e Humanas em Saúde da ABRASCO.

As evidências revelaram que $18,3 \%(\mathrm{~N}=23)$ dos trabalhos foram realizados por meio de parcerias, contemplando autores oriundos das três modalidades institucionais classificadas nesse estudo (Tabela 4). A Academia foi a principal indutora dos trabalhos em parceria, atuando com os "Serviços" e "ONGs", ao passo que a interface entre essas duas últimas instituições foi encontrada em apenas um trabalho. Não obstante, a diversidade dos atores que atuam no campo da Saúde Coletiva brasileira, cabe ponderar que nos congressos da ABRASCO, predomina a participação de profissionais vinculados às universidades públicas e privadas e aos institutos públicos de pesquisa.

No presente estudo, dentre os 12 temas classificados sobre o "Terceiro Setor na Saúde", aferiu-se que a Academia investigou quase a totalidade dos temas (11 temas), seguida pelos "Serviços", responsáveis por 7 temas e em menor número pelas "ONGs" com 5 temas, conforme demonstra a Tabela 4. Mais de um terço dos trabalhos conduzidos pela Academia priorizaram em ordem 
decrescente a análise sobre os movimentos sociais e a participação na saúde, o controle social nos conselhos de saúde e a atuação das ONGs na área da saúde. Causa espécie que menos de $4 \%$ dos objetos abordados pela Academia no âmbito do Terceiro Setor, referiram-se à avaliação de projetos e ações programáticas ou à produção de conhecimento científico, uma vez que essas são funções estratégicas e consonantes à missão das instituições universitárias. Essa questão evidencia por um lado, a emergência na universidade do tema "Terceiro Setor na Saúde" e, por outro lado, a potencialidade dessa temática nas agendas de pesquisa, com vistas a adensar conhecimento técnicocientífico sobre a dinâmica do "Terceiro Setor" e seus impactos na implementação das políticas e das práticas de saúde pública.

Nesse prisma, ao problematizar o papel da Academia como um dos lócus privilegiados de produção de saberes e de intervenções voltadas à mitigação das iniquidades sociais, concordamos com Gohn (2014) ao postular que o

compromisso social é uma construção histórica entre diferentes agentes e atores sociais. (...) É fruto de um feixe de relações e ações, com sentido e significado inteligível e pactuado entre as instituições e organizações envolvidas - quer seja uma universidade ou outra, onde há aprendizagens, construção de saberes, ações que situem os indivíduos no centro do processo e não as imagens de uma empresa, a busca do lucro ou do marketing. (...) Consequentemente, a universidade é um dos palcos privilegiados destas ações. Que saberes impulsiona ou constrói? Qual seu papel no processo de mudança e transformação social, especialmente em contextos de desigualdades e injustiças sociais? (Gohn, 2014:86).

Em relação aos "Serviços", os principais temas elencados referiram-se à intersetorialidade e redes (25\%), seguido dos temas, mix público-privado na saúde, avaliação de projetos ou ações programáticas e educação em saúde, perfazendo 16,7\% cada. Apesar do crescimento observado nos arranjos institucionais operados via administração indireta, por meio da contratação de Organizações Sociais da Saúde em vários estados e municípios brasileiros, somente um trabalho oriundo da modalidade "Serviços", abordou essa questão relevante para a compreensão das formas de organização e gestão no SUS. Na mesma perspectiva, foi encontrado somente um trabalho sobre os temas, vigilância em saúde e controle social nos conselhos, o que causa espécie considerando a natureza e a missão das instituições tipificadas na modalidade "Serviços" (Tabela 4).

Em relação às ONGs, o principal tema abordado discutiu a inserção e atuação dessas instituições no âmbito da saúde, correspondendo a $60 \%$ dos trabalhos apresentados nessa modalidade (Tabela 4). Chama a atenção que os temas relacionados às ações de advocacy e incidência política, os quais tradicionalmente tendem a assumir destaque na agenda das ONGs que atuam no setor Saúde, assim como o exercício do controle social nos conselhos e o direito à saúde, além do voluntariado, não foram temáticas incluídas na produção dessas entidades da sociedade civil. Por fim, na modalidade das parcerias, os temas mais elencados foram os movimentos sociais e a participação na saúde, assim como o desenvolvimento das ações de educação em saúde, responsáveis por $43,4 \%$ dos trabalhos. Os temas menos frequentes, com apenas um resumo identificado trataram do direito à saúde e da atuação das ONGs na Saúde. 
Tabela 4. Frequência absoluta e relativa dos temas sobre o "Terceiro Setor na Saúde" apresentados no I, II, III, IV e V Congressos Brasileiros de Ciências Sociais e Humanas em Saúde, segundo o vínculo institucional dos autores. ABRASCO, 1995 - 2011.

\begin{tabular}{|c|c|c|c|c|c|c|c|c|c|c|}
\hline \multirow[t]{2}{*}{ Temas } & \multicolumn{2}{|c|}{ Academia } & \multicolumn{2}{|c|}{ Serviços } & \multicolumn{2}{|c|}{ ONG } & \multicolumn{2}{|c|}{ Parcerias } & \multicolumn{2}{|c|}{ Total } \\
\hline & $\mathbf{N}$ & $\%$ & $\mathbf{N}$ & $\%$ & $\mathbf{N}$ & $\%$ & $\mathbf{N}$ & $\%$ & $\mathbf{N}$ & $\%$ \\
\hline Movimentos sociais e participação na saúde & 13 & 16,0 & 0 & 0 & 1 & 10,0 & 5 & 21,7 & 19 & 15,1 \\
\hline Controle social nos conselhos de saúde & 12 & 14,8 & 1 & 8,3 & 0 & 0 & 0 & 0 & 13 & 10,3 \\
\hline Direito à saúde & 4 & 4,9 & 0 & 0 & 0 & 0 & 1 & 4,3 & 5 & 4,0 \\
\hline Atuação ONG na Saúde & 11 & 13,6 & 0 & 0 & 6 & 60,0 & 1 & 4,3 & 18 & 14,3 \\
\hline Voluntariado & 6 & 7,4 & 0 & 0 & 0 & 0 & 0 & 0 & 6 & 4,8 \\
\hline Gestão/Gerência nas Organizações Sociais de Saúde & 7 & 8,6 & 1 & 8,3 & 1 & 10,0 & 0 & 0 & 9 & 7,1 \\
\hline Mix público-privado & 3 & 3,7 & 2 & 16,7 & 1 & 10,0 & 4 & 17,4 & 10 & 7,9 \\
\hline Intersetorialidade e redes & 10 & 12,3 & 3 & 25,0 & 0 & 0 & 0 & 0 & 13 & 10,3 \\
\hline Educação em saúde & 9 & 11,1 & 2 & 16,7 & 1 & 10,0 & 5 & 21,7 & 17 & 13,5 \\
\hline Avaliação de projetos ou ações programáticas & 3 & 3,7 & 2 & 16,7 & 0 & 0 & 3 & 13,0 & 8 & 6,3 \\
\hline Produção de conhecimento teórico/metodológico & 3 & 3,7 & 0 & 0 & 0 & 0 & 4 & 17,4 & 7 & 5,6 \\
\hline Vigilância em saúde & 0 & 0 & 1 & 8,3 & 0 & 0 & 0 & 0 & 1 & 0,8 \\
\hline Total & 81 & 100 & 12 & 100 & 10 & 100 & 23 & 100 & 126 & 100 \\
\hline
\end{tabular}

Fonte: Os autores

Em todas as instituições analisadas, verificou-se que a principal abordagem metodológica adotada no desenvolvimento das pesquisas ou dos relatos de experiências foi a qualitativa, correspondendo a $80,8 \%$ da produção, conforme a Tabela 5. A segunda abordagem metodológica mais utilizada foi a quantitativa, perfazendo $11,2 \%$, seguida das metodologias mistas com 8,0\%. Esses achados são consonantes ao perfil da produção científica no subcampo das Ciências Sociais e Humanas em Saúde, no qual predomina a utilização de técnicas qualitativas variadas para investigar a complexidade dos problemas de saúde, a partir da vertente marxista da determinação social do processo saúde-doença (Luz \& Mattos, 2010).

Constatou-se que os trabalhos realizados em parceria contemplaram todas as possibilidades metodológicas, ao passo que a totalidade da produção encontrada nos "Serviços" foi ancorada na metodologia qualitativa (Tabela 5). Especificamente, entre as entidades da sociedade civil, predominou o emprego de técnicas qualitativas, perfazendo $80 \%$ e em menor proporção, o uso de técnicas mistas (20\%). Na modalidade das parcerias, induzida, sobretudo pela Academia, excetuandose a abordagem qualitativa, verificou-se que as técnicas quantitativas foram mais frequentes na condução dos trabalhos alcançando $26,1 \%$, comparada às metodologias mistas com apenas $4,3 \%$. Cabe ressaltar que o uso da abordagem mista empregada na análise dos objetos de pesquisa e temas elencados pelos investigadores, pode ser considerado um fenômeno recente e provavelmente associado à maior circulação de saberes interdisciplinares no campo da Saúde Coletiva (Nunes e cols., 2010).

Por outro lado, Deslandes e Maksud (2020) ao examinarem a formação em metodologia no subcampo das Ciências Sociais e Humanas em Saúde nos programas de Pós-Graduação em Saúde Coletiva, entre 2002 e 2016, encontraram duas modalidades de disciplinas: as multidisciplinares que 
discutem principalmente os métodos epidemiológicos e qualitativos e as disciplinas específicas que abordam os vários métodos empregados nesse subcampo. Segundo as autoras, foram oferecidas ao todo 963 disciplinas sobre métodos, entretanto, apesar da constância na oferta das disciplinas, poucas abordaram as metodologias em Ciências Sociais e também foi exígua a indicação de leitura dos autores clássicos nas referências bibliográficas das ementas. As pesquisadoras identificaram que "(...) Karl Marx recebeu apenas 48 citações nos 15 anos analisados. Émile Durkheim recebeu apenas 37 citações e Max Weber somente 25 citações, ocupando a $134^{\mathrm{a}}$ posição dentre os autores citados" (Deslandes \& Maksud, 2020:8).

Nesse sentido, ao discutir a sustentabilidade da interdisciplinaridade, Ianni (2018) ratifica a dinâmica do campo da Saúde Coletiva e adverte que "a perda da centralidade das ciências sociais no campo decorre de inúmeros fatores (...), por exemplo, a grande transformação havida no conjunto do campo, o seu crescimento, desdobramentos, evoluções, diversidades, multiplicidades temáticas e de interesse. Paradoxalmente, essa perda de centralidade decorre também de certa perda do compromisso original das ciências sociais em saúde com questões de método (Ianni, 2018:110).

Tabela 5. Frequência absoluta e relativa dos temas sobre o "Terceiro Setor na Saúde" apresentados pelas instituições no I, II, III, IV e V Congressos Brasileiros de Ciências Sociais e Humanas em Saúde, segundo a metodologia. ABRASCO, 1995 - 2011.

\begin{tabular}{lcccccccccc}
\hline & \multicolumn{2}{c}{ Academia } & \multicolumn{2}{c}{ Serviços } & \multicolumn{2}{c}{ ONG } & \multicolumn{2}{c}{ Parcerias } & \multicolumn{2}{c}{ Total } \\
Metodologia & $\mathbf{N}$ & $\mathbf{\%}$ & $\mathbf{N}$ & $\mathbf{\%}$ & $\mathbf{N}$ & $\mathbf{\%}$ & $\mathbf{N}$ & $\mathbf{\%}$ & $\mathbf{N}$ & $\mathbf{\%}$ \\
\hline Qualitativa & 65 & 81,3 & 12 & 100 & 8 & 80,0 & 16 & 69,6 & 101 & 80,8 \\
Quantitativa & 8 & 10,0 & 0 & 0,0 & 0 & 0,0 & 6 & 26,1 & 14 & 11,2 \\
Quanti/Quali & 7 & 8,8 & 0 & 0,0 & 2 & 20,0 & 1 & 4,3 & 10 & 8,0 \\
& & & & & & & & & & \\
\hline Total & $\mathbf{8 0}$ & $\mathbf{1 0 0}$ & $\mathbf{1 2}$ & $\mathbf{1 0 0}$ & $\mathbf{1 0}$ & $\mathbf{1 0 0}$ & $\mathbf{2 3}$ & $\mathbf{1 0 0}$ & $\mathbf{1 2 5}$ & $\mathbf{1 0 0}$ \\
\hline
\end{tabular}

Fonte: Anais dos Congressos ABRASCO.

Obs.: Não foi identificada a metodologia de um trabalho.

Na Tabela 6, que mostra as várias modalidades de apresentação nos Congressos, verificou-se que $41,3 \%$ dos trabalhos foram apresentados na modalidade oral e $16,7 \%$ por meio de pôsteres, demonstrando a visibilidade do tema emergente "Terceiro Setor na Saúde" no subcampo das Ciências Sociais e Humanas em Saúde. Cabe elucidar que, na última edição, a ABRASCO incorporou duas modalidades novas para a socialização dos trabalhos, a saber: o pôster eletrônico, apresentado oralmente pelo expositor, porém com um tempo menor de exposição em relação a modalidade oral; e a publicação nos Anais, a qual não contemplou a apresentação oral durante a programação oficial do congresso. Essas duas modalidades foram responsáveis por respectivamente, $22,2 \%$ e $19,8 \%$ dos resumos apresentados no V Congresso. 
Tabela 6. Frequência absoluta e relativa dos temas sobre o "Terceiro Setor na Saúde" apresentados pelas instituições no I, II, III, IV e V Congressos Brasileiros de Ciências Sociais e Humanas em Saúde, segundo a modalidade de apresentação. ABRACO 1995 - 2011.

\begin{tabular}{lcccccccccc}
\hline & \multicolumn{2}{c}{ Academia } & \multicolumn{2}{c}{ Serviços } & \multicolumn{2}{c}{ ONG } & \multicolumn{2}{c}{ Parcerias } & \multicolumn{2}{c}{ Total } \\
Modalidade & $\mathbf{N}$ & $\mathbf{\%}$ & $\mathbf{N}$ & $\mathbf{\%}$ & $\mathbf{N}$ & $\mathbf{\%}$ & $\mathbf{N}$ & $\mathbf{\%}$ & $\mathbf{N}$ & $\mathbf{\%}$ \\
\hline Oral & 35 & 43,2 & 3 & 25,0 & 2 & 20,0 & 12 & 52,2 & 52 & 41,3 \\
Pôster & 15 & 18,5 & 4 & 33,3 & 2 & 20,0 & 0 & 0,0 & 21 & 16,7 \\
Pôster eletrônico & 15 & 18,5 & 2 & 16,7 & 4 & 40,0 & 7 & 30,4 & 28 & 22,2 \\
Publicação Anais & 16 & 19,8 & 3 & 25,0 & 2 & 20,0 & 4 & 17,4 & 25 & 19,8 \\
\hline Total & $\mathbf{8 1}$ & $\mathbf{1 0 0}$ & $\mathbf{1 2}$ & $\mathbf{1 0 0}$ & $\mathbf{1 0}$ & $\mathbf{1 0 0}$ & $\mathbf{2 3}$ & $\mathbf{1 0 0}$ & $\mathbf{1 2 6}$ & $\mathbf{1 0 0}$ \\
\hline
\end{tabular}

Fonte: Anais dos Congressos ABRASCO.

Nota-se que nos trabalhos oriundos da Academia, destacou-se, sobretudo a modalidade oral perfazendo 43,2\% das apresentações (Tabela 6). Nos "Serviços", um terço das apresentações foram pôsteres e entre as ONGs, verificou-se a menor proporção de trabalhos orais (20\%). Quanto aos trabalhos em parceria, mais da metade das apresentações foram orais, seguidas de pôster eletrônico. Pode-se afirmar que o tema do "Terceiro Setor na Saúde" obteve visibilidade na programação dos congressos promovidos pela ABRASCO, especialmente nas apresentações do tipo oral "tradicional" ou por pôster eletrônico, totalizando $63,5 \%$ do universo analisado.

\section{Considerações Finais}

A análise sobre o "Terceiro Setor na Saúde", baseada nos trabalhos dos congressos da ABRASCO, evidenciou que a visibilidade dessa temática no subcampo das Ciências Sociais e Humanas em Saúde, ocorreu em consonância às mudanças observadas na implementação das políticas de saúde no Brasil durante a década de 1990. Constatamos, na produção científica dessa temática, o protagonismo das organizações da sociedade civil, tipificadas como ONGs, bem como dos movimentos populares de saúde, porém as abordagens sobre o voluntariado na saúde e o direito à saúde seguiram sendo exíguas.

Majoritariamente, os estudos e os relatos de experiências foram conduzidos por profissionais vinculados a Academia e/ou Institutos Públicos de Pesquisa, mas também houve a participação de profissionais de "Serviços" (unidades de saúde, secretarias estaduais/municipais de saúde e Ministério da Saúde) e de "ONGs" (incluindo os movimentos sociais e movimentos populares de saúde), assim como as parcerias firmadas entre essas modalidades institucionais. Do ponto de vista do desenho e da 
seleção dos materiais e métodos de investigação, verificou-se o uso das três abordagens metodológicas, porém predominam os trabalhos ancorados na metodologia qualitativa, em consonância a maioria das pesquisas conduzidas sobre os problemas de saúde nesse subcampo da Saúde Coletiva. Em relação à dinâmica dos congressos, a maioria dos trabalhos foi apresentado por meio da modalidade oral, seguida da apresentação por pôsteres, indicando assim, uma posição de destaque na programação desses eventos.

Dentre os efeitos da consolidação do "Terceiro Setor na Saúde", ressaltamos o avanço dos arranjos institucionais voltados à terceirização e à privatização das instituições de saúde, ancorados no arcabouço jurídico da Reforma do Aparelho do Estado Brasileiro na década de 1990 e, induzidos, sobretudo a partir da edição das Lei Federais que criaram as Organizações Sociais de Saúde (OSS) e as Organizações da Sociedade Civil de Interesse Público (OSCIP), entre outras figuras jurídicas (Brasil, 1995; Brasil, 1998; Brasil, 1999). A partir desse marco legal, a gerência dos equipamentos de saúde foi delegada para administração indireta, porém com processos de controle e de regulação pelos entes públicos, ainda considerados deficientes quanto ao monitoramento e avaliação dos resultados alcançados na melhoria dos indicadores de saúde da população. Não obstante a relevância dessa questão, no presente estudo, somente $15,1 \%$ dos trabalhos analisados, abordaram temas como o mix público-privado na saúde e a gestão/gerência das Organizações Sociais da Saúde. Recomenda-se assim, a incorporação e manutenção desses temas estratégicos para a gestão do SUS nas agendas de pesquisa em curso nos subcampos das Ciências Sociais e Humanas em Saúde e das Políticas, Planejamento e Gestão em Saúde.

Constatamos que, no esquadrinhamento dos temas, ganhou relevo no contexto do "Terceiro Setor", a importância da Atenção Básica no SUS, sobretudo quanto à discussão das redes intersetoriais e da educação em saúde. Em um estudo anterior sobre o tema em questão, Barboza e colaboradores (2015) aferiram que 47,6\% dos resumos analisados, versaram sobre aspectos relacionados à implementação da rede de Atenção Básica no território sanitário para o aperfeiçoamento das políticas públicas de saúde.

No período recente, após o golpe de 2016 no Brasil, a Atenção Primária em Saúde (APS) vem sofrendo constantes ameaças de privatização, especialmente no governo Jair Bolsonaro em vigência. $\mathrm{O}$ "Projeto Previne" editado em 2020 pelo Ministério da Saúde, induz ao processo de privatização, fragilizando a organização e o financiamento da oferta da saúde integral e equânime na lógica territorial. Portanto, postulamos que a privatização não é compatível ao direito à saúde, consonante à Constituição Federal de 1988, solapando assim, o arcabouço ético-legal do SUS, expresso na garantia do acesso universal à saúde integral. Assim advogamos, uma APS forte, integral e equânime em todas regiões de saúde do país, operando como coordenadora do sistema de saúde, com vistas a assegurar a longitudinalidade do cuidado (Starfield, 2004).

O enfrentamento dessas questões em particular e da própria complexidade do "Terceiro Setor na Saúde", reafirmam o papel estratégico do exercício do controle social nos conselhos de saúde, tema que ao longo das cinco edições analisadas, perdeu visibilidade nos congressos do subcampo das Ciências Sociais e Humanas em Saúde. Na contemporaneidade, a participação social materializa uma das principais invenções do SUS, devendo ser repolitizada à luz do ideário da Reforma Sanitária Brasileira, ancorada nos pilares da justiça social e da determinação social do processo saúde-doença. Portanto, a pluralidade de atores e instituições que compõe o SUS em sua capilaridade são corresponsáveis pelo aperfeiçoamento e sustentabilidade desse braço do sistema de proteção social. 


\section{Referências Bibliográficas}

Barboza, Renato., \& Lopes, Tarsio Vinicius. (2009). Organizações não governamentais na região da Amazônia legal brasileira: o caso da saúde. Psicologia Política, 9(17), 149-169. Acessado em 31 de Março de 2020, de http://pepsic.bvsalud.org/scielo.php?script=sci arttext\&pid=S1519549X2009000100010\&lng=pt\&tlng=pt.

Barboza, Renato., Ianni, Aurea Maria Zöllner., Alves, Olga Sofia Fabergé., Cintra, João Pedro Scholl., \& Souza, Fernanda Kaliane Martins. (2014). Análise da produção científica brasileira sobre o tema AIDS no campo das Ciências Sociais e Humanas em Saúde Coletiva. Patrimonio: Economía Cultural y Educación para la Paz (MEC-EDUPAZ), 1, 448-451.

Barboza, Renato., Ianni, Áurea Maria Zöllner., Alves, Olga Sofia Fabergé., Rocha, Ane Talita da Silva., \& Viana, Sabrina Daniela Lopes. (2015). Terceiro Setor na Saúde: a produção científica dos Congressos Brasileiros de Ciências Sociais e Humanas em Saúde na ABRASCO. Journal of Management and Primary Health Care, 6(1), 18-35. Acessado em 6 de Abril de 2020, de https://doi.org/10.14295/jmphc.v6i1.235

Barboza, Renato., Dantas, Jean Carlos de Oliveira., Cervantes, Vilma., Silva, Alessandro Soares da., Alves, Olga Sofia Fabergé., \& Santos, Rani B. C. E. (2017). Desafios para sustentabilidade da prevenção às DST/Aids nos projetos de Organizações da Sociedade Civil no estado de São Paulo. BEPA: Boletim Epidemiológico Paulista, 14(162), 17-31. Acessado em 31 de Março de 2020, de: https://docs.bvsalud.org/biblioref/ses-sp/2017/ses-36291/ses-36291-6501.pdf

Brasil. (1995). Plano Diretor da Reforma do Aparelho do Estado. Brasília: Câmara da Reforma do Estado.

Brasil. (2011). Política Nacional de Atenção Básica. Brasília: Ministério da Saúde.

Brasil. (19 de Setembro de 1990a). Lei no 8.080. Dispõe sobre as condições para a promoção, proteção e recuperação da saúde, a organização e o funcionamento dos serviços correspondentes e dá outras providências. Diário Oficial da União, 19/09/1990.

Brasil. (28 de Dezembro de 1990b). Lei no 8.142. Dispõe sobre a participação da comunidade na gestão do Sistema Único de Saúde e sobre as transferências intergovernamentais de recursos financeiros na área da saúde e dá outras providências. Diário Oficial da União, 28/12/1990.

Brasil. (15 de Maio de 1998). Lei $n^{\circ}$ 9.637. Dispõe sobre a qualificação de entidades como organizações sociais, a criação do Programa Nacional de Publicização, a extinção dos órgãos e entidades que menciona e a absorção de suas atividades por organizações sociais, e dá outras providências. Diário Oficial da União, 18/05/1998.

Brasil. (23 de Março de 1999). Lei $n^{\circ}$ 9.790. Dispõe sobre a qualificação de pessoas jurídicas de direito privado, sem fins lucrativos, como Organizações da Sociedade Civil de Interesse Público, institui e disciplina o Termo de Parceria, e dá outras providências. Diário Oficial da União, 24/03/1999.

Bresser-Pereira. Luiz Carlos. (2011). Reforma gerencial do Estado, teoria política e ensino da administração pública. Revista Gestão \& Políticas Públicas, 1(2):1-6. Acessado em 31 de março de 2020, de: https://www.revistas.usp.br/rgpp/article/view/97836 
Buss, Paulo Marchiori. (2003). Uma introdução ao conceito de promoção da saúde. Em Dina Czeresnia, \& Carlos Machado de Freitas. (Eds.). Promoção da Saúde: conceitos, reflexões, tendências, (pp. 15-38). Rio de Janeiro: Fiocruz.

Canesqui, Ana Maria. (1998). Ciências Sociais e Saúde no Brasil: Três Décadas de Ensino e Pesquisa. Ciência \& Saúde Coletiva, 3(1), 131-168.

Canesqui, Ana Maria. (2008). As Ciências Sociais e Humanas em Saúde na Associação Brasileira de Pós-graduação em Saúde Coletiva. Physis, 18(2), 215-250.

Deslandes, Suely, \& Maksud, Ivia. (2020). Ensino de metodologias em Ciências Sociais e Humanas nos programas de pós-graduação em Saúde Coletiva (2002-2016). Cadernos de Saúde Pública, 36(5), e00133619. Acessado em 31 de Março de 2020, de: https://doi.org/10.1590/0102$311 \times 00133619$

Falconer, Andres Pablo. (1999). A Promessa do Terceiro Setor: Um estudo sobre a construção do papel das Organizações Sem fins Lucrativos e de seu campo de gestão. Dissertação (Mestrado). Universidade de São Paulo, São Paulo.

Ferrari, Junia, Oliveira, Laís, Torres, Marina, \& Soalheiro, Maria. (2019). Dos Movimentos Sociais às Organizações da Sociedade Civil: repensando o associativismo no contexto neoliberal, In XVIII Encontro Nacional da ANPUR, 1-17. Natal. UFRN. Acessado em 31 de Março de 2020, de: http://anpur.org.br/xviiienanpur/anaisadmin/capapdf.php?reqid=800

Godoy-Flores, Iván. (2017). Dos Movimentos Sociais às Políticas Públicas: contribuições da Psicologia Política. Revista Gestão \& Políticas Públicas, 7(2), 195-201. Acessado em 27 de maio de 2019, de: https://doi.org/10.11606/rg\&pp.v7i2.174541

Godoy-Flores, Iván. (2019). Uma Epistemologia das Políticas Públicas: elementos para a ação pública. Revista Gestão \& Políticas Públicas, 9(2), 354-360. Acessado em 27 de maio de 2019, de: https://doi.org/10.11606/rgpp.v9i2.183251

Gohn, Maria da Glória. (2000). Mídia, terceiro setor e MST: impacto sobre o futuro das cidades e do campo. Petrópolis: Vozes.

Heimann, Luiza Sterman., Ibanhes, Lauro César., \& Barboza, Renato. (Org.). (2005). O Público e o Privado na Saúde. São Paulo: Hucitec.

Ianni, Áurea Maria Zöllner., Barboza, Renato., Alves, Olga Sofia Fabergé., Rocha, Ane Talita da Silva.,Viana, Sabrina Daniela Lopes., \& Torres, Renan. (2012). Questões contemporâneas nas Ciências Sociais em Saúde: o estudo de temas emergentes nos congressos brasileiros de Ciências Sociais e Humanas em Saúde, ABRASCO 1995 - 2007. Relatório final de pesquisa CNPq 4703892009-5.

Ianni, Áurea Maria Zöllner., Spadacio, Cristiane., Barboza, Renato., Alves, Olga Sofia Fabergé., Viana, Sabrina Daniela Lopes., \& Rocha, Ane Talita da Silva. (2014). Trajetórias profissionais na constituição das Ciências Sociais e Humanas em Saúde na Abrasco. Physis, 24, 1315-1336.

Ianni, Áurea Maria Zöllner., Spadacio, Cristiane., Barboza, Renato., Alves, Olga Sofia Fabergé., Viana, Sabrina Daniela Lopes., \& Rocha, Ane Talita da Silva. (2015). Os Congressos Brasileiros de Ciências Sociais e Humanas em Saúde da ABRASCO: um campo científico em disputa. Ciência \& Saúde Coletiva, 20(2), 503-513.

Ianni, Áurea Maria Zoolner. (2018). Mudanças sociais contemporâneas e saúde: estudo sobre a teoria social e saúde pública no Brasil. São Paulo: Hucitec. 
Instituto Brasileiro de Geografia e Estatística (IBGE). (2012). As fundações privadas e associações sem fins lucrativos no Brasil 2010. Rio de Janeiro: IBGE.

Instituto Brasileiro de Geografia e Estatística (IBGE). (2019). As fundações privadas e associações sem fins lucrativos no Brasil: 2016. Rio de Janeiro: IBGE.

Luz, Madel Therezinha, \& Mattos, Rafael da Silva. (2010). Dimensões qualitativas na produção científica, tecnológica e na inovação em Saúde Coletiva. Ciência \& Saúde Coletiva, 15(4), 19451953.

Marsiglia, Regina Maria Giffoni., Spinelli, Selma Patti., Lopes, Marina França., \& Silva, Thiago Carvalho Pelucio. (2003). Das ciências sociais para as ciências sociais em saúde: a produção científica de pós-graduação em ciências sociais. Ciência \& Saúde Coletiva, 8(1), 275-285.

Minayo, Maria Cecília de Souza. (2004). O desafio do conhecimento: pesquisa qualitativa em saúde. São Paulo, Rio de Janeiro: Hucitec/Abrasco.

Minayo, Maria Cecília de Souza. (2013). A produção de conhecimentos na interface entre as Ciências Sociais e Humanas e a Saúde Coletiva. Saúde e Sociedade, 22(1), 21-31.

Montaño, Carlos. (2010). Terceiro setor e questão social: crítica ao padrão emergente de intervenção social. São Paulo: Cortez.

Nunes, Everardo Duarte. (2006). A trajetória das ciências sociais em saúde na América Latina: revisão da produção científica. Revista de Saúde Pública, 40(Nº esp.), 64-72.

Nunes, Everardo Duarte., Ferreto, Lirane Elize., Oliveira, Ana Luiza de Oliveira e., Nascimento, Juliana Luporini do., Barros, Nelson Filice de., \& Castellanos, Marcelo Eduardo Pfeiffer. (2010). O campo da Saúde Coletiva na perspectiva das disciplinas. Ciência \& Saúde Coletiva, 15(4), 19171922.

Paim, Jairnilson Silva., \& Almeida Filho, Naomar de. (2000). A crise da Saúde Pública e a utopia da Saúde Coletiva. Salvador: Casa da Qualidade.

Paim, Jairnilson Silva. (2008). Reforma sanitária brasileira: contribuição para a compreensão e crítica. Salvador: Edufba, Rio de Janeiro: Fiocruz.

Ribeiro, Patrícia Tavares., \& Castro, Leonardo. (2019). Ciências Sociais em saúde: perspectivas e desafios para a saúde coletiva. Saúde Debate, 43 (7 n. Especial), 165-178.

Silva, Alessandro Soares da. (2007). O processo de onguização do Estado e dos movimentos sociais e o seu impacto no âmbito das políticas públicas. In XIV Encontro Nacional da ABRAPSO, 1-12. Rio de Janeiro: ABRAPSO. Acessado em 31 de Março de 2020, de: http://www.abrapso.org.br/siteprincipal/anexos/AnaisXIVENA/conteudo/pdf/trab_completo_87.pd $\underline{\mathrm{f}}$

Silva, Alessandro Soares da. (2009). Contribuições dos Movimentos Sociais para a Desprivatização da Ética na Perspectiva da Psicologia Política. Flávia Mori Sarti, \& Gislene Aparecida dos Santos. (Org.). Ética, Pesquisa e Políticas Públicas. São Paulo: Rubio. Acessado em 19 de maio de 2019, de:

https://www.researchgate.net/publication/344041671 Contribuicoes dos Movimentos Sociais pa ra_a_Desprivatizacao_da_Etica_na_perspectiva_da_Psicologia_Politica

Silva, Alessandro Soares da. (2012). Psicologia Política, movimentos sociais e políticas públicas. Tese de Livre Docencia. Universidade de São Paulo, São Paulo.

Silva, Alessandro Soares da. (2013). Um enfoque psicopolítico das políticas públicas. Revista Electrónica de Psicología Política, 11(30), 1-6. Acessado em 12 de maio de 2019, de: 
https://www.researchgate.net/publication/344664075 Um Enfoque Psicopolitico das Politicas P ublicas_Un_Enfoque_Psicopolitico_de_las_Politicas_Publicas

Silva, Alessandro Soares da. (2015). Garantias de direitos e de dignidade na construção do humano. Psicologia Política, 15(32), 11-16. Acessado em 11 de abril de 2020, de: http://pepsic.bvsalud.org/scielo.php?script=sci arttext\&pid=S1519549X2015000100001\&lng=pt\&tlng=pt.

Silva, Alessandro (2018a). A Ação Pública: um outro olhar sobre Estado, Sociedade e Políticas Públicas. Revista Gestão \& Políticas Públicas, 8(1), 194-204. Acessado em 17 de abril de 2019, de: https://www.revistas.usp.br/rgpp/article/view/175154

Silva, Alessandro S. (2018b). Um Esboço do que poderia ser a Psicologia Política da Ação Pública. Cadernos da ANPEPP, GT 62, Psicologia Política. Acessado em 25 de fevereiro de 2018, de: https://www.researchgate.net/publication/344134091_Um_Esboco_do_que_poderia_ser_a_Psicolo gia_Politica_da_Acao_Publica

Starfield, Barbara. (2004). Atenção Primária: equilíbrio entre necessidades de saúde, serviços e tecnologia. Brasília: UNESCO/Ministério da Saúde.

Stralen, Cornelis Johannes van. (1996). The Struggle over a National Health Care System; the 'movimento sanitário'and health policy-making in Brazil. Utrecht: FSW/RUU.

Recebido em 07/02/2020.

Revisado em 03/04/20.

Aceito 18/07/2020. 